\title{
A Case of Abdominothoracic Cystic Lymphangiomatosis Presenting as Left-Sided Inguinal Swelling in a Young Adult: Radiological Manifestation
}

\author{
Dibyamohan Hazarika $\quad$ Abhinav Aggarwal2, \\ ${ }^{1}$ Department of Diagnostic \& Interventional Radiology, Ayursundra \\ Superspecialty Hospital, Guwahati, Assam, India \\ 2Department of Radiology, Mata Chanan Devi Hospital, New Delhi, \\ Delhi, India
}

\begin{abstract}
Address for correspondence Abhinav Aggarwal, MBBS, MD, Department of Radiology, Mata Chanan Devi Hospital, C-1, Janakpuri, New Delhi 110058, Delhi, India

(e-mail: abhinavagg@gmail.com; dr_dibyamohan@yahoo.co.in).
\end{abstract}

J Gastrointestinal Abdominal Radiol ISGAR 2021;4:70-73.

\author{
Abstract \\ Keywords \\ - cystic \\ lymphangiomatosis \\ $-M R I$ \\ $-\mathrm{CT}$ \\ - intervention radiology
}

Lymphatic system imaging has been a challenging field in Radiology. We here describe a case of a 26-year-old male who presented with complaints of left-sided inguinoscrotal swelling and was subsequently diagnosed with abdomino-thoracic cystic lymphangiomatosis. Detailed imaging evaluation across the modalities of ultrasonography, computed tomography scan and magnetic resonance imaging evaluation revealed extensive involvement and diagnostic imaging appearance of the unique lymphatic system abnormality. Imaging findings were confirmed on histopathology.

\section{Introduction}

Cystic lymphangiomatosis is defined as diffuse cystic dilatation of the lymphatic system characterized by multiorgan involvement. ${ }^{1-3}$ The organs commonly affected apart from the lymphatic system are the lungs, liver, spleen, bone, and skin. It is primarily seen in the pediatric age group since it is believed to develop from maldevelopment of the lymphatic systems in intrauterine life. ${ }^{2-4}$ Around 65 to $90 \%$ of cystic lymphangiomas are diagnosed by the age of 2 years, and the central nervous system is never affected by the pathology. ${ }^{2,5}$ The lymphangiomas are composed of lymphatic endothelium-lined chylous-filled cystic spaces. Cavernous and capillary hemangiomas and cystic hygromas account for the primary differential diagnosis, which can be differentiated based on imaging and histopathological features. ${ }^{1,3}$ In cases of involvement of solid organ parenchyma, prognosis is generally poor. ${ }^{6}$ We here describe a case of a 26 -year-old male who presented with complaints of left-sided inguinoscrotal swelling and was diagnosed with cystic lymphangiomatosis. Imaging findings were confirmed on histopathology.

\section{Case Report}

A 26-year-old male presented with complaints of left-sided inguinoscrotal swelling and pain in the inguinoscrotal region radiating to the groin since the last two months. After clinical evaluation, ultrasonography (USG) examination of the whole abdomen and bilateral inguinoscrotal regions was performed. USG revealed multiple well-defined cystic lesions in the left inguinal region; however, no evidence of hydrocele was noted ( - Fig. 1). Color Doppler images did not show any flow in the lesions.

The patient was then advised computed tomography (CT) scan of the abdomen and thorax. Contrast-enhanced CT (CECT) of the abdomen was performed in noncontrast and postcontrast venous phases after intravenous (IV) administration of $80 \mathrm{~mL}$ of iodinated contrast (Ultravist 370, Bayer AG, Berlin, Germany) at the rate of $3.5 \mathrm{~mL} /$ second in a 128-slice MDCT scanner (Siemens Definition AS, Erlangen, Germany). CT scan was performed using protocols to reduce radiation including autodose modulation. CECT revealed multiple confluent cystic lesions in the retroperitoneum, from the published online

November 10, 2020
DOI https://doi.org/

$10.1055 / \mathrm{s}-0040-1718248$

ISSN 2581-9933.
(C2020. Indian Society of Gastrointestinal and Abdominal Radiology. This is an open access article published by Thieme under the terms of the Creative Commons Attribution-NonDerivative-NonCommercial-License, permitting copying and reproduction so long as the original work is given appropriate credit. Contents may not be used for commercial purposes, or adapted, remixed, transformed or built upon. (https://creativecommons.org/licenses/by-nc-nd/4.0/).

Thieme Medical and Scientific Publishers Pvt. Ltd. A-12, 2nd Floor, Sector 2, Noida-201301 UP, India 


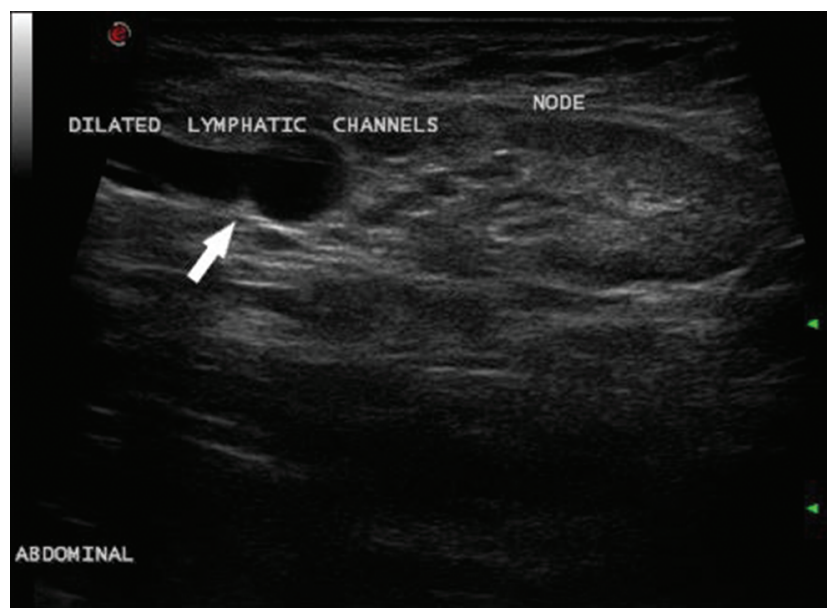

Fig. 1 High-resolution ultrasonography image reveals dilated lymphatics (arrow) reaching up to normal sized lymph nodes in the left inguinal region.

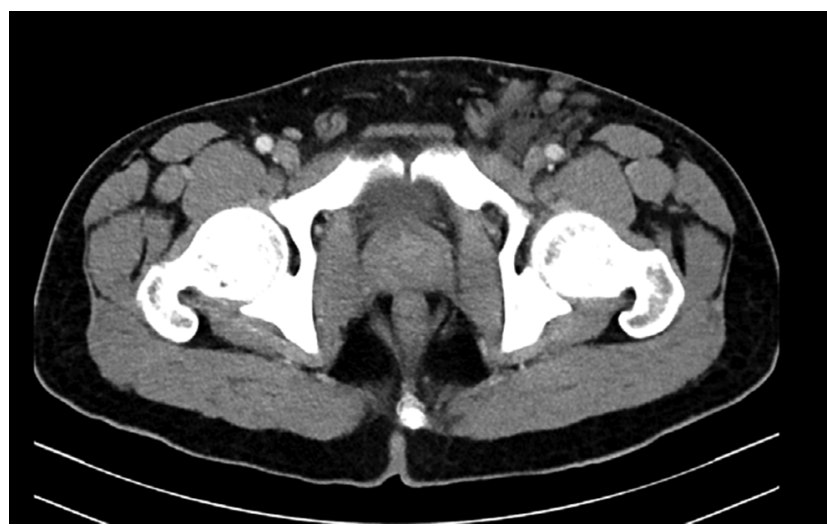

Fig. 2 Axial postcontrast computed tomography scan image showing multiple hypodense confluent cystic lesions in the left inguinal region.

infradiaphragmatic region till aortic bifurcation extending to left the ileoinguinal region (-Figs. 2-5). Based on these CT scan findings, the patient underwent guided fluid aspiration and biopsy of the left inguinal component for cytological/ histopathological studies, which revealed neutrophils and lymphoid tissue in a hemorrhagic background. No atypical cells were seen. Serum CA19-9 (carbohydrate antigen 19-9) and LDH (lactate dehydrogenase) were also within normal limits.

To study the extent of disease and ascertain the magnetic resonance (MR) imaging characteristics of the disease, magnetic resonance imaging (MRI) examination of the abdomen as well as the thorax was performed. MRI of the thorax was performed in our 1.5-T MRI scanner (Magnetom Avanto TIM, Erlangen, Germany) for delineation of the mediastinal component of lymphatic abnormality in standard multiplanar sequences including IV gadolinium contrast (MultiHance, Bracco, Singen, Germany) injection at the dose of $0.1 \mathrm{mmol} /$ $\mathrm{kg}$ body weight. Along with the already known findings, MR study also revealed the communication of the cystic lesions with cisterna chyli and dilated thoracic duct in the abdomen

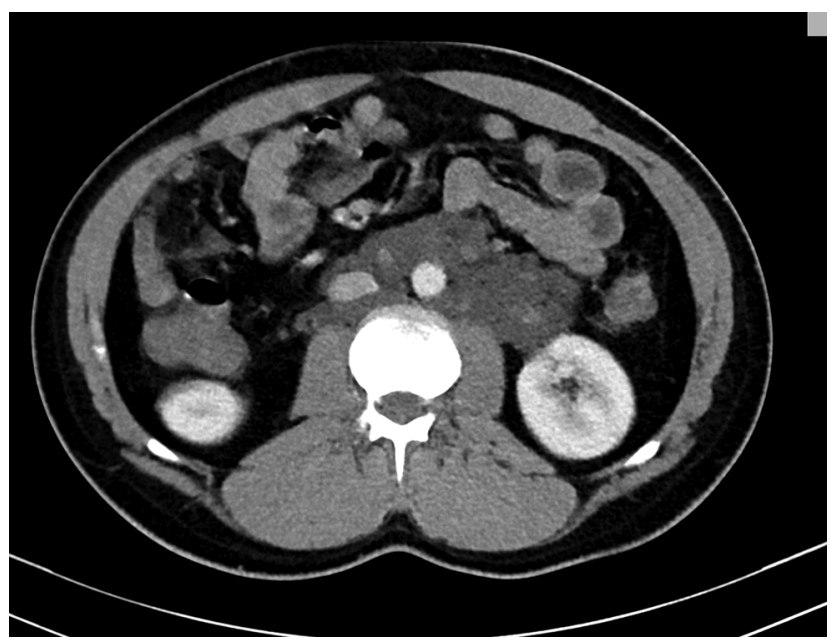

Fig. 3 Axial postcontrast computed tomography scan image showing confluent hypodense cystic lesions in the retroperitoneum encasing abdominal aorta and inferior vena cava. There was no hydronephrosis.

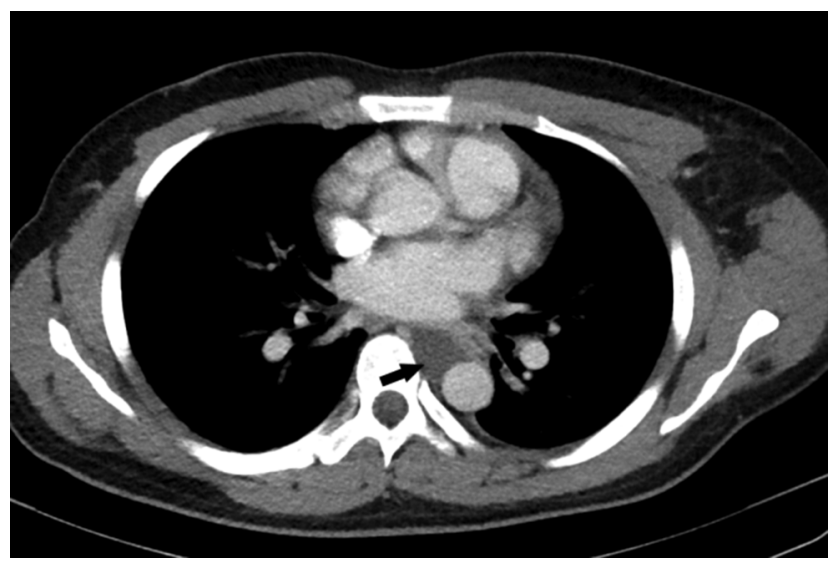

Fig. 4 Axial postcontrast computed tomography scan image showing a fluid attenuation linear tubelike structure (arrow) along the anatomical location of the thoracic duct in the left paraesophageal region.

and thorax, respectively (-Figs. 6-9). Histopathology was performed to confirm our diagnosis ( - Fig. 10).

We did not find any bony lesions or involvement of any solid parenchymal organ such as the liver or spleen. The patient was counseled regarding the nature of the disease and given option of limited surgical resection. However, the patient opted for symptomatic treatment only.

\section{Discussion}

Cystic lymphangiomatosis was first described by Rodenber in $1828 .^{7}$ It is thought to originate from the maldevelopment of the lymphatic system during the 14th to 20th week of intrauterine life. These are rare benign lesions consisting of eosinophilic homogeneous material or chyle., ${ }^{1,2}$ The imaging features of generalized cystic lymphangiomatosis (GCL) have been compared with those of other complex lymphatic abnormalities such as Gorham-Stout's disease (GSD) and 


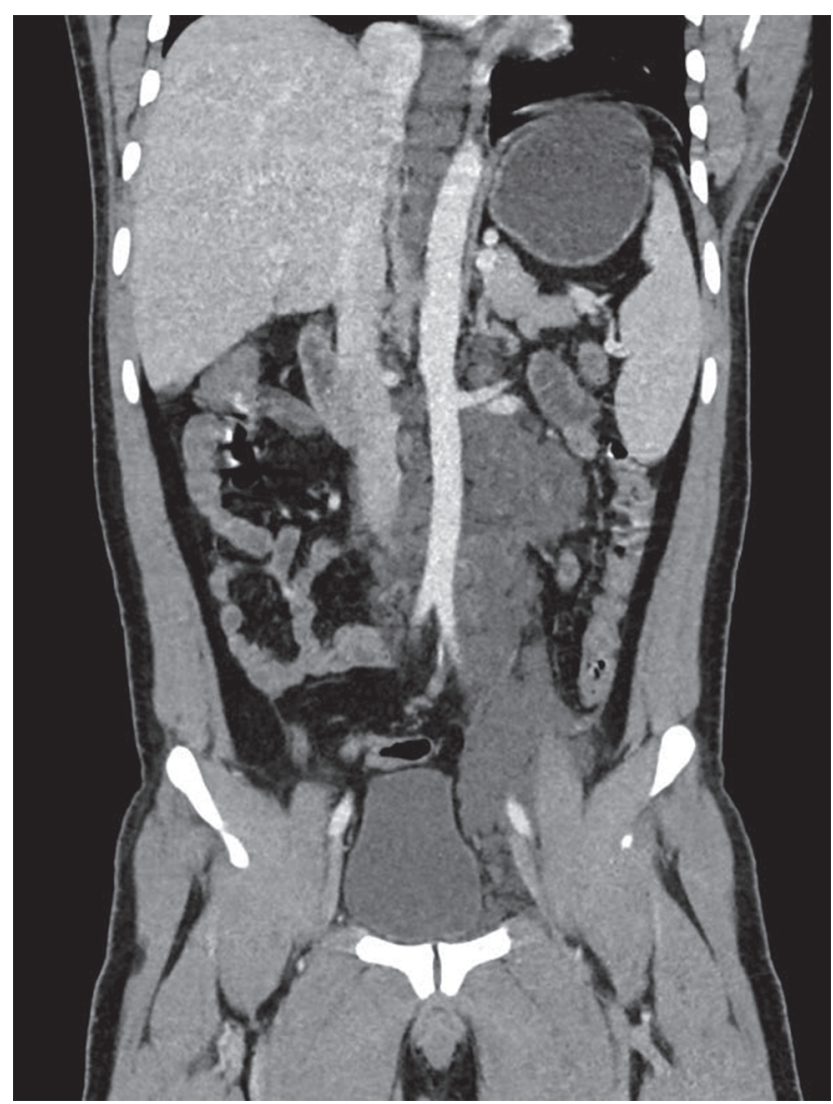

Fig. 5 Abdomen contrast-enhanced computed tomography image with curved multiplanar reconstruction showing communication of the left inguinal component with the retroperitoneal component.

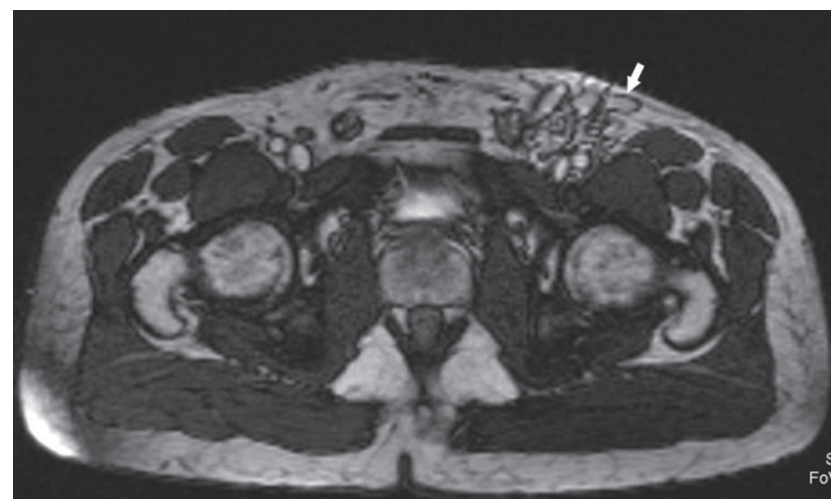

Fig. 6 Axial T2-weighted image showing confluent mass of thin-walled cystic structures (arrow) in the left inguinal region.
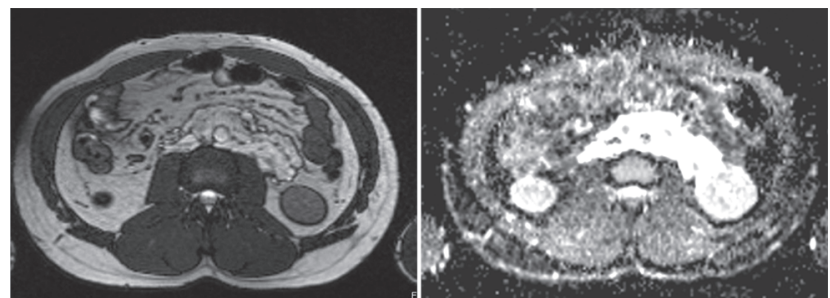

Fig. 7 Axial T2-weighted image showing cystic hyperintense lesions in the retroperitoneum with corresponding apparent diffusion coefficient image showing no restriction on diffusion.
Kaposiform lymphangiomatosis (KLA). GSD and KLA have widespread bone involvement and are therefore differentiated based on the imaging features of bony lytic lesions, whereas GCL has more soft tissue involvement. ${ }^{6}$ Since histological features of various lymphatic abnormalities are overlapping, correlation with imaging findings is mandatory. ${ }^{1,3,4}$

Clinically, patients may be asymptomatic, but symptoms depend on the extent of organ involvement. Bone and pleural/ lung involvement can lead to fractures and respiratory distress, respectively. The presence of chyle in pleural or

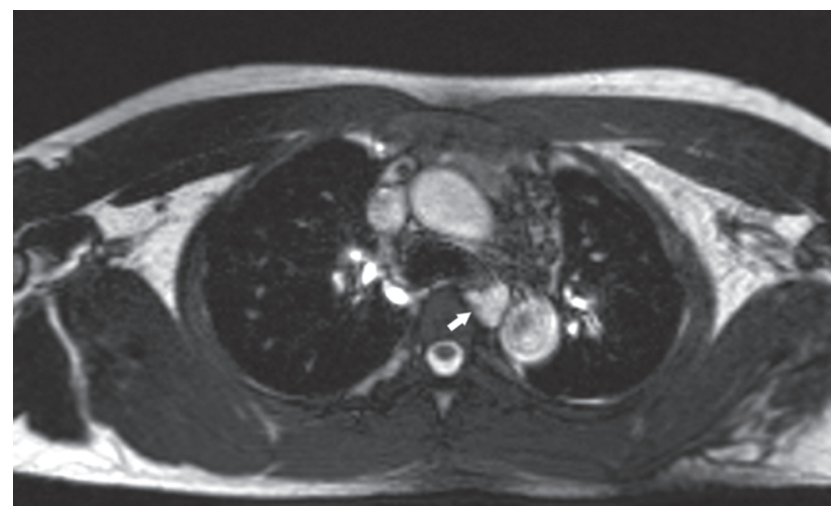

Fig. 8 Axial T2-weighted image showing elongated cystic hyperintense structure (arrow) in the mediastinum along the left paraesophageal region. On serial sections, it was continuing from the retroperitoneum and was seen to reach left the internal jugular vein and subclavian junction, consistent with a dilated thoracic duct.

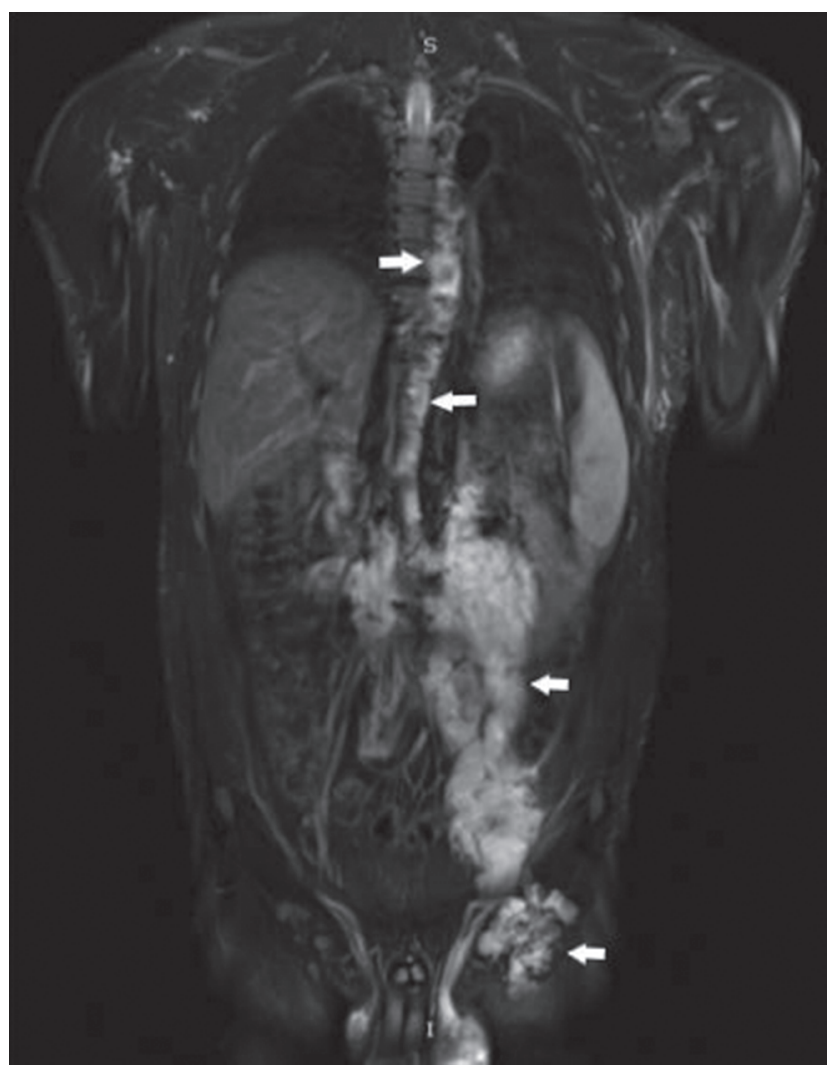

Fig. 9 Coronal STIR MRI image showing a T2 hyperintense thinly septated lobulated lesion (arrows) in the retroperitoneum communicating superiorly with the dilated thoracic duct and inferiorly with a similar component in the left inguinal region. 


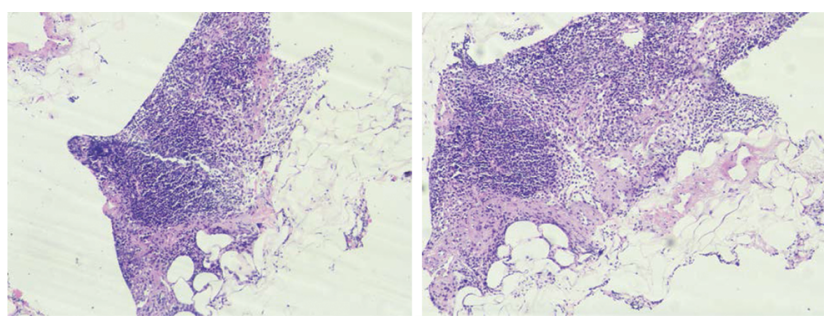

Fig. 10 Histological section from biopsy specimen of inguinal swelling showing dilated lymphatic channels lined by flattened endothelial cells. Wall showed lymphocytes and lymphoid follicles (H\&E, 40X).

pericardial effusion is usually a sign of poor prognosis. ${ }^{3}$ In our case, the patient presented with swelling in the left inguinoscrotal region. On imaging examination, we found that the patient had cystic lesions extending all across the retroperitoneum and mediastinum. Although $45 \%$ of the patients present with bony or solid organ lesions, we did not find any organ or bone involvement in our case. Involvement of the axial and appendicular skeleton is the most commonly reported finding in the literature. Bony lymphangiomas are commonly confused with bony hemangiomas; however, in isolated cases, their management does not differ. ${ }^{5}$

Although mediastinal involvement has been reported in the pediatric population, it is more commonly seen in adults. ${ }^{1}$ With regard to the presently available literature, the most striking feature of our case is the age of the patient. We could find only one article describing imaging and histopathological features of GCL in the third decade of life. ${ }^{8}$ Considering that mediastinal involvement is associated with progression/more severe disease, our patient was relatively asymptomatic. ${ }^{3}$

Various studies have implicated the corroborative role of imaging studies along with histopathology for diagnosis of the disease. ${ }^{1-4,9}$ On imaging evaluation by USG, cystic lymphangiomatosis appears as confluent anechoic spaces with thick branching septations in the involved compartment. There is no color flow on Doppler interrogation. They appear as hypodense septated cystic spaces with $\mathrm{CT}$ attenuation values ranging from 5 to $10 \mathrm{HU}$ on noncontrast CT. There is usually mass effect with no postcontrast enhancement on both CT and MRI. On MRI, lesions appear as confluent lobulated T2 hyperintense cystic spaces with no diffusion restriction. They may communicate with dilated cisterna chyli or thoracic duct or nearby lymph nodes. In our case, in addition to assisting in diagnosis and defining the extent of disease, we were also able to demonstrate the communication between cystic lesions and cisterna chyli and thoracic duct in retroperitoneum and mediastinum, respectively.
Management of lymphangiomas includes surgical excision for localized lesions, whereas palliative procedures such as pericardial and pleural fluid drainage are used for generalized lymphangiomatosis. Radiation therapy and interferon-alfa have also shown to be effective. ${ }^{8}$

We would like to conclude by saying that with the advances in imaging, more unusual presentation of this entity is being seen. This is one of the very few descriptions of findings of GCL in an adult patient.

\section{Authors' Contributions}

Both the authors contributed equally to the article and approved it for submission.

\section{Funding \\ None.}

\section{Conflict of Interest}

None declared.

\section{References}

1 Wunderbaldinger P, Paya K, Partik B, et al. CT and MR imaging of generalized cystic lymphangiomatosis in pediatric patients. AJR Am J Roentgenol 2000;174(3):827-832

2 Ozel A, Uysal E, Dokucu AI, Erturk SM, Basak M, Cantisani V. US, CT and MRI findings in a case of diffuse lymphangiomatosis and cystic hygroma. J Ultrasound 2008;11(1):22-25

3 Yang DH, Goo HW. Generalized lymphangiomatosis: radiologic findings in three pediatric patients. Korean J Radiol 2006;7(4):287-291

4 Wong CS, Chu TYC. Clinical and radiological features of generalised lymphangiomatosis. Hong Kong Med J 2008;14(5):402-404

5 El-Essawy MT, Hassan HH. Cystic hygroma of the neck with multiple bone lymphangiomas. Egyp J Radiol Nuc Med 2011;42(2):153-155

6 Joshi M, Phansalkar DS, Simple lymphangioma to generalized lymphatic anomaly: role of imaging in disclosure of a rare and morbid disease. Case Rep Radiol 2015;2015:603859

7 Rao BK, AuBuchon J, Lieberman LM, Polcyn RE. Cystic lymphangiomatosis of the spleen: a radiologic-pathologic correlation. Radiology 1981;141(3):781-782

8 Marom EM, Moran CA, Munden RF. Generalized lymphangiomatosis. AJR Am J Roentgenol 2004;182(4):1068

9 Rasalkar DD, Chu WC. Generalized cystic lymphangiomatosis. Pediatr Radiol 2010;40(1, Suppl 1) :S47 